\title{
Role of LASP-1, a novel SOX9 transcriptional target, in the progression of lung cancer
}

\author{
JIANGUANG SHI, JING GUO and XINJIAN LI
}

\begin{abstract}
Department of Thoracic Surgery, Ningbo First Hospital, Haishu, Ningbo, Zhejiang 315010, P.R. China
\end{abstract}
Received April 11, 2017; Accepted August 7, 2017

DOI: 10.3892/ijo.2017.4201

\begin{abstract}
Lung cancer accounts for most cancer-related deaths worldwide. However, the underlying mechanism by which it mediates the progression of lung cancer remains unclear. Expression of LASP-1 (LIM and SH3 protein 1) was evaluated in lung cancer tissues and tumor-adjacent normal tissues using immunohistochemistry and western blotting. Functional studies have shown that siRNA-mediated silencing of LASP-1 in human lung cancer cells and reduced cell proliferation, migration, and invasion. Flow cytometry and immunofluorescence staining also revealed that rate of cell apoptosis was increased after knockdown of expression of LASP-1, thereby suggesting that LASP-1 may function as an oncogene during lung cancer progression. SOX9 is an important transcription factor, which is involved in the development of several types of human cancer. Further analysis has showed the presence of a consensus-binding site of SOX9 in the promoter region of LASP-1. Mechanistic investigations showed that LASP-1 was transcriptionally activated by SOX9. Through luciferase reporter and ChIP assays, we demonstrated that LASP-1 was a direct target gene of sex determining region Y-box 9 (SOX9). Knockdown of SOX9 expression by RNA interference reduces cell proliferation and induces apoptosis of lung cancer cells, which was consistent with the results obtained from silencing the expression of LASP-1 in NCI-H1650 cells. Together, these findings indicated that LASP-1, as a downstream target of SOX9, may act as a novel biomarker for lung cancer and plays an important role in cell proliferation, migration, and invasion.
\end{abstract}

\section{Introduction}

Lung cancer is the leading cause of cancer-related deaths worldwide, accounting for $12.7 \%$ of new cancer cases and $18.2 \%$ of new cancer deaths $(1,2)$. Lung cancer is a major health concern and pose heavy burden on family and society (2). Based on

Correspondence to: Professor Xinjian Li, Department of Thoracic Surgery, Ningbo First Hospital, 59 Liuting Street, Haishu, Ningbo, Zhejiang 315010, P.R. China

E-mail: nbfh_ljx@hotmail.com

Key words: lung cancer, LASP-1, SOX9, tanscriptional activation histology, lung cancer can be classified into non-small cell lung cancer (NSCLC) and small cell lung cancer (SCLC) (3). In general, NSCLC accounts for $>80 \%$ of all lung cancers, and can be further classified into squamous carcinoma, adenocarcinoma, and large cell carcinoma (4,5). Despite the significant improvement in surgery and chemotherapy, the prognosis of advanced lung cancer remains poor $(6,7)$. The overall 5-year survival of lung cancer remains $\sim 17 \%$ (8). Tumor recurrence and distant metastasis are the leading causes of death in advanced lung cancer patients (9). Thus, it is important to understand the underlying molecular mechanism of tumor progression and metastasis in order to design new therapeutic agents for lung cancer patients.

LIM and SH3 protein 1 (LASP-1), also called metastatic lymph node gene 50 proteins (MLN50), were identified from a cDNA library of breast cancer $(7,10)$. LASP-1 encodes a putative protein of 261 amino acids, with an N-terminal LIM domain and a C-terminal SRC homology 3 (SH3) domain $(10,11)$. The LIM domain is followed by two actin-binding domains in the core of LASP-1 protein, which interacts with various binding partners within the cytoskeleton and transmit signals from the cytoplasm into the nucleus $(11,12)$. The SH3 domain is involved in protein-protein interactions, binding proline-rich sequences, such as zyxin and pallidin (13). Owing to its ubiquitous expression in many tissues, LASP-1 exhibits wide range of biological functions, including cell morphology, signal transduction, and cell motility $(14,15)$. LASP-1 has been reported to be overexpressed in several types of human cancers, such as breast cancer $(16,17)$, ovarian cancer (18), gastric cancer (19), hepatocellular cancer (20), colorectal cancer (21), lung cancer (7,22), and renal cell cancer (23), suggesting LASP-1 as a potential biomarker for the treatment of cancer. Several studies have shown that LASP-1 can promote cell proliferation, migration, and invasion in a wide variety of tumors both in vitro and in vivo $(11,17)$. In this study, we demonstrated the role of LASP-1 as potential biomarker in cancer; however, the underlying mechanism of how LASP-1 mediates oncogenesis in lung cancer remains unclear (7).

SOX9, a transcription factor and member of the SOX family, is a key regulatory protein, which is involved in developmental processes, including male sex determination, chondrogenesis, neurogenesis, and neural crest development (24-26). Recent cogent evidence has provided a link between SOX9 and cancer progression. SOX9 overexpression has been reported in breast cancer (27), prostate cancer (28), colorectal cancer (29), and 
lung cancer (30-32), where its expression is correlated with malignancy and overall survival. Although an association between upregulation of SOX9 and lung cancer progression has been reported (33), the potential regulatory effect of SOX9 and LASP-1 in lung cancer needs further validation.

In the present study, we investigated the expression of LASP-1 in lung cancer using human tissue samples and assessed the potential role of LASP-1 in lung cancer cell-lines. The results have shown that knockdown of LASP-1 expression by siRNA reduced cell proliferation and increased cell apoptosis. Moreover, mechanistic investigation showed that LASP-1 was a critical downstream target of SOX9. Taken together, these findings suggested that SOX9-LASP1 axis plays an important role in cell proliferation, migration, and invasion.

\section{Materials and methods}

Ethics statement. The Institutional Ethics Committee at the Ningbo First Hospital, China, approved this study, and written informed consent was obtained from all the patients prior to their participation.

Cell culture. Human lung cancer cell lines, A549, NCI-H838, NCI-H1299, and NCI-H1650 were obtained from the American Type Culture Collection (ATCC, Rockville, MD, USA) and cultured in RPMI-1640 (Hyclone) supplemented with 10\% fetal bovine serum (FBS) (Gibco) and 1\% penicillin/streptomycin. The immortalized human bronchial epithelial cell line, BEAS-2B (ATCC), was maintained in BEGM medium (Lonza, Switzerland) supplemented with $10 \% \mathrm{FBS}$ and $1 \%$ penicillin/streptomycin. The cells were cultured at $37^{\circ} \mathrm{C}$ in water-saturated $5 \% \mathrm{CO}_{2}$ atmosphere.

Tissue samples. Thirteen human lung cancer and paired tumoradjacent normal tissues were obtained from Ningbo First Hospital, China. All patients were pathologically and clinically diagnosed as lung cancer patients. None of the patients had undergone radiotherapy or chemotherapy before surgical resection. The histological diagnosis of melanoma was evaluated according to the World Health Organization (WHO). The tissues, $>5 \mathrm{~cm}$ away from the cancer lesions, were defined as tumor-adjacent normal tissues.

Plasmid. Twist2, Nkx2-5, and Sox9 were amplified using PCR from NCI-1650 and cloned into the pGL3-basic vector (Promega, Madison, WI, USA). Mutations of the Twist2, Nkx2-5, and Sox9 binding site was performed by Quik Change Site Mutagenesis kit (Stratagene, La Jolla, CA, USA) according to the manufacturer's instructions. The primers used for cloning are as follows: Twist2 KpnI: 5'-GGGGTACCTCTGA ACACAATGATTGGGT-3' (forward), Twist2 XhoI: 5'-CCCTC GAGGAAGTTCACAGGGCAGAGTC-3' (reverse); Twist2 mut: 5'-CCCGGGTGGCAGATCAGTTCTAACTCATTGTC ATTCAACA-3' (forward), Twist2 mut: 5'-TGTTGAATGACA ATGAGTTAGAACTGATCTGCCACCCGGG-3' (reverse); Nkx2-5 KpnI: 5'-GGGGTACCATAAAACATTCATTA GGCTCC-3' (forward), Nkx2-5 XhoI: 5'-CCCTCGAGTCGTA TCTATGGAAAGGGTAT-3' (reverse); Nkx2-5 mut: 5'-TAGT TAGGAAAAAATGATAACCCGTTCTTTTTTGTGTAC-3' (forward), Nkx2-5 mut: 5'-GTACACAAAAAAGAACGG GTTATCATTTTTTCCTAACTA-3' (reverse); Sox9 KpnI: 5'-GGGGTACCCAATCTTAGACAAATCACCA-3' (forward), Sox9XhoI:5'-CCCTCGAGGCTAGTCTTGAACTTCTGGT-3' (reverse); Sox9 mut: 5'-TCAATTCCATACAAATGTCACAG GCTGAATGTATATGGC-3' (forward), Sox9 mut: 5'-GCC ATATACATTCAGCCTGTGACATTTGTATGGAATTGA-3' (reverse).

Immunohistochemistry (IHC). The immunohistochemical analysis was performed to determine the expression of LASP-1 protein in 13 pairs of human lung cancer and paired non-tumor tissues, using avidin-biotin-peroxidase complex method, with anti LASP-1 antibody. The tissue sections were incubated with polyclonal antibody against LASP-1 (1:200; Millipore, USA) overnight at $4^{\circ} \mathrm{C}$.

RNA extraction and real-time PCR. Total RNA was extracted using TRIzol regent (Invitrogen) and reverse transcribed using the transcriptase cDNA synthesis kit (Takara, Dalian, China) according to the manufacturer's instructions. Real-time PCR analysis was performed using SYBR-Green (Takara) in ABI 7500 fast fluorescence temperature cycler. The primers were used at a concentration of $0.5 \mu \mathrm{M}$ to generate single PCR product. The primers used are as follows: LASP-1: sense, 5'-GGT GCGGCAAGATCGTGTA-3'; antisense, 5'-TGCAGGTCTC GCAATGGAA-3'. GAPDH sense, 5'-ACGGATTTGGTCGT ATTGGG-3'; antisense,5'-CGCTCCTGGAAGATGGTGAT-3'.

Small interfering RNAs. Small interfering RNAs (siRNA) were used to knockdown the expression of LASP-1 and SOX9 in lung cancer cells. All the siRNA duplexes were purchased from GenePharma (GenePharma, Shanghai, China). The specific siRNAs (siLASP-1: 5'-TGTAGTTCTTCATGTTCA GTG-3' and siSOX9: 5'-TCTTCATGAAG GGGTCCAGGA-3') were used for RNA interference and the non-specific scramble siRNA duplexes (5'-TTCTCCGAACGTGTCACGTTT-3') were used as normal control. The siRNA duplexes were transduced into NCI-H1650 cells at a final concentration of $50 \mathrm{nM}$ using Lipofectamine ${ }^{\mathrm{TM}} 2000$ (Invitrogen Corp., Carlsbad, CA, USA).

Cell proliferation. The cells were seeded in 96-well plates, and the proliferation of the cells was assayed at $0,24,48$, and $72 \mathrm{~h}$ using CCK-8 kit (Dojindo Laboratories, Japan) according to the manufacturer's instructions. Cell viability was assessed by measuring of absorbance at $450 \mathrm{~nm}$ using a microplate reader.

Cell migration and invasion assays. The cell migration ability was determined using wound healing assay. The migration was assessed by determining the movement of cells into a scraped area created by pipette tip. After scratching, the cells were cultured in media supplemented with $0.1 \%$ FBS to eliminate the effect of cell proliferation. The cell invasive ability of lung cancer cells was determined using Transwell chamber $(8 \mu \mathrm{m}$, Corning). Cells $\left(5 \times 10^{4}\right)$ in serum-free media were placed in the top chambers, and complete media was added to the bottom chambers. The chambers were incubated for $24 \mathrm{~h}$ at $37^{\circ} \mathrm{C}$. After incubation, the medium was removed from both the 
Table I. Patient characteristics.

\begin{tabular}{|c|c|}
\hline Clinical features & No. $(\%)$ \\
\hline Age, average (range, years) & $70(48-91)$ \\
\hline Tumor size, average (range, $\mathrm{cm}$ ) & $3.2(1-7)$ \\
\hline \multicolumn{2}{|l|}{ Sex } \\
\hline Male & $7(70)$ \\
\hline Female & $3(30)$ \\
\hline \multicolumn{2}{|l|}{ Cigarette } \\
\hline Yes & $8(80)$ \\
\hline No & $2(20)$ \\
\hline \multicolumn{2}{|l|}{ pT stage } \\
\hline $\mathrm{T} 1-\mathrm{T} 2$ & 5 \\
\hline $\mathrm{T} 3-\mathrm{T} 4$ & 5 \\
\hline \multicolumn{2}{|l|}{ Histology } \\
\hline Squamous & 5 \\
\hline Adenoma & 5 \\
\hline \multicolumn{2}{|l|}{ Metastasis status } \\
\hline Negative & 6 \\
\hline Positive & 4 \\
\hline \multicolumn{2}{|l|}{ Pro-operation radiation } \\
\hline Yes & 2 \\
\hline No & 8 \\
\hline \multicolumn{2}{|l|}{ Lasp-1 expression (IHC) } \\
\hline Negative & 0 \\
\hline Weak & 2 \\
\hline Moderate & 5 \\
\hline Strong & 3 \\
\hline
\end{tabular}

wells, and the chambers were fixed with methanol for $30 \mathrm{~min}$ and stained with crystal violet for $30 \mathrm{~min}$.

Flow cytometry. Cell apoptosis was evaluated using FITCAnnexin V Apoptosis Detection kit. Briefly, the cells were harvested and washed with cold PBS, and then incubated with $5 \mu \mathrm{l}$ of FITC-conjugated Annexin V and $5 \mu \mathrm{l}$ PI for $10 \mathrm{~min}$ at room temperature in the dark. The samples were analyzed by flow cytometry. For cell cycle assay, the cells were harvested and fixed in $70 \%$ ethanol for $48 \mathrm{~h}$. The nuclei were stained with $50 \mu \mathrm{g} / \mathrm{ml}$ PI in $1 \%$ Triton X-100 containing $100 \mu \mathrm{g} / \mathrm{ml}$ DNase-free RNase, and the DNA content was analyzed by flow cytometry.

Luciferase reporter assays. The LASP-1 promoter region $-2,500 /+1$ construct was amplified from genomic DNA of NCI-H1650 cells. The WT and mutated LASP-1 promoter constructs were cloned into the pGL3-Basic reporter gene vector and verified by sequencing. HEK293T cells were transfected by Lipofectamine 2000 (Invitrogen) in 6-well plates. Co-transfection of Renilla luciferase plasmid was used as the internal control for transfection efficiency. Luciferase activities were measured using the Dual luciferase assay kit (cat. no. E1960, Promega), with a Berthold Detection system $\mathrm{GmbH}$ chemiluminometer. The results were expressed as ratio of firefly luciferase activity to Renilla luciferase activity.
Chromatin immunoprecipitation (ChIP). ChIP was performed according to the manufacturer's instructions (Active Motif, Carlsbad, CA, USA) in NCI-H1650 cells, with anti-SOX9 antibody. Real-time PCR analysis was performed using SYBR-Green (Takara) in ABI 7500 fast fluorescence temperature cycler. Normal rabbit IgG was used as negative control. ChIP-qPCR assay was performed in triplicates. The primers used for ChIP are as follows: LASP-1-F, AAGCTACCTTGG CCAGTCG; R, AGGGGTCTCACTATGTTGCC.

Statistical analysis. Student's t-test or one-way analysis of variance (ANOVA) was used to evaluate the significance between groups, using the statistical package SPSS 17.0 in all the experiments. A value of $\mathrm{P}<0.05$ was considered to indicate statistical significance.

\section{Results}

LASP-1 expression in lung cancer. Increasing evidence has showed that expression of LASP-1 is increased in various human cancers, such as breast cancer. To elucidate the expression level of LASP-1 in lung cancer, we compared LASP-1 expression in 13 lung cancer tissue samples and adjacent normal tissues. Patient characteristics, such as age, sex, histopathological diagnosis, clinical stage, and metastasis status are shown in Table I. Real-time PCR and IHC were performed to determine SOX9 and LASP-1 expression in tissues. As shown in Fig. 1A, SOX9 expression was measured by IHC staining, and elevated expression presented in tumor tissues than normal. The results showed that expression of LASP-1 was increased in lung cancer tissues as compared to normal tissues (Fig. 1B and C). To confirm the increase in expression, western blotting was performed to determine LASP-1 expression level in lung cancer tissue and paired tumor-adjacent normal tissue samples. As illustrated in Fig. 1D, LASP-1 expression was upregulated in tumor tissues as compared to normal tissues. Next, we determined the expression of LASP-1 in lung cancer cell lines. As shown in Fig. 1E, LASP-1 expression was increased in lung cancer cell-lines, NCI-H1299 and NCI-H1650, compared with LASP-1 expression level in immortalized human bronchial epithelial BEAS-2B cells.

Functional analysis after knockdown of LASP-1 expression. Based on our findings that LASP-1 was overexpressed in lung cancer cells (Fig. 1), we utilized siRNAs approach to knockdown LASP-1 expression in lung cancer NCI-H1650 cells. Western blot assay was performed to determine the expression level of LASP-1. The results showed that LASP-1 was significantly silenced by siRNAs (Fig. 2A). siRNA2 was selected for further experiments. The cell proliferation ability of LASP-1 was determined using CCK- 8 assay after knockdown of the LASP-1 expression. The results showed that cell proliferation was significantly inhibited at 24, 48, and $72 \mathrm{~h}$ in LASP-1 knockdown cells as compared to control cells (Fig. 2B). The role of LASP-1 in the regulation of cell migration and invasion was studied by wound healing assay, using reconstituted extracellular matrices in porous culture chambers. As shown in Fig. 2C and D, wound closure occurred gradually after scratching in control cells, whereas this effect was significantly reduced in siLASP-1 cells. In line 
A
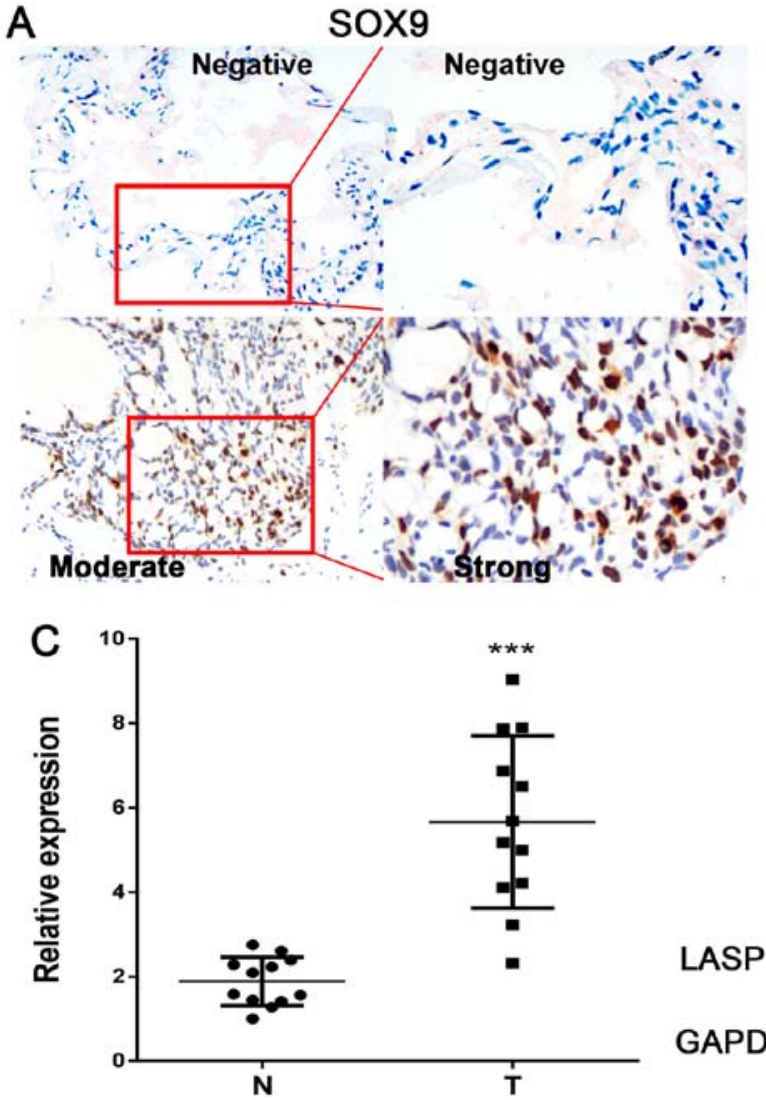

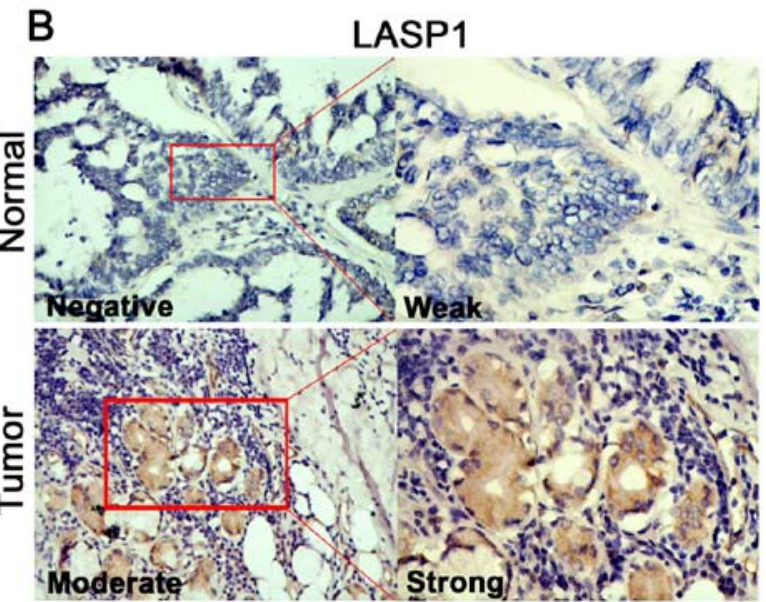

$\mathrm{D}$

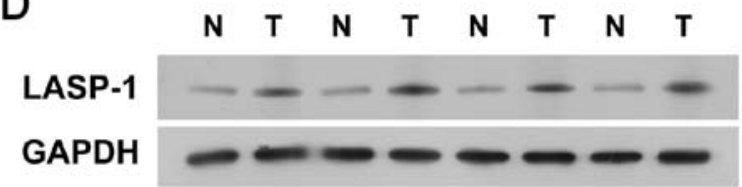

$\mathrm{E}$

BEAS-2B A549 NCl-H838 NCl-H1299 NCl-H1650

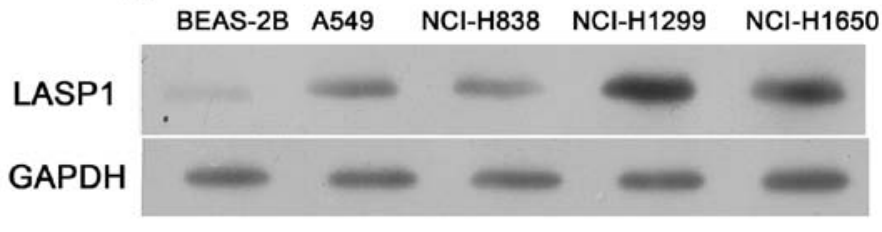

Figure 1. Expression level of LASP-1 in lung cancer tissues and cell lines. (A) Immunohistochemistry analysis of SOX9 protein levels in 13 lung cancer tissues. Representative microscopic images of normal and tumor tissues stained with anti-SOX9 antibody (B) Immunohistochemistry analysis of LASP-1 protein levels in 13 lung cancer tissues. Representative microscopic images of normal and tumor tissues stained with an anti-LASP-1 antibody; the staining intensity is represented as negative, weak, moderate and strong, respectively. Brown color indicates LASP-1 positivity. No IHC staining in lung cancer for LASP-1 protein $(0+$, negative); faint IHC membrane staining in $>10 \%$ tumor cells (1+, weak); moderate complete IHC membrane staining in $>10 \%$ tumor cells $(2+$, moderate); strong complete IHC membrane staining in $>10 \%$ tumor cells (3+, strong). The red squares indicate LASP-1 expression in same position. (C) Real-time PCR indicated that LASP-1 levels were significantly upregulated in 13 paired lung cancer tissues as compared with adjacent non-cancerous tissues. (D) Western blot analysis revealed that LASP-1 was upregulated in cancer tissues $(n=4)$ compared with the normal control $(n=4)$. (E) Western blot analysis of endogenous LASP-1 in lung cancer cell lines.

with this finding, siLASP-1 cells showed reduced invasive capacity as compared to control cells (Fig. 2E). Cell apoptosis was determined by staining the cells for Annexin V and PI. siRNA-mediated silencing of LASP-1 led to cell apoptosis of lung cancer cells (Fig. 2F), based on the observations of the percentage of the Annexin V-positive cells (increase from 9.2 to $17.1 \%$ in NCI-1650). It was also observed that the proportion of cells in S phase was decreased, whereas the proportion of cells in G1 phase was significantly increased as compared to control (Fig. 2G). Moreover, immunofluorescence images of lung cancer cells transfected with siRNA and stained with antibody against LASP-1. The results showed that LASP-1 expression was decreased in NCI-1650 cells transfected with LASP-1 siRNAs as compared to NC group (Fig. 3A). Hoechst staining also showed that cell apoptosis was increased after knockdown of the expression of LASP-1 in NCI-1650 cells (Fig. 3B), which was consistent with the results obtained from Annexin V and PI staining (Fig. 2F). Together, these results suggested that LASP-1 may function as an oncogene in lung cancer progression.

LASP-1 is a direct target gene of SOX9. Although the function of LASP-1 in cancer progression has been well demonstrated, little is known about the underlying molecular mechanism of LASP-1 in mediating the progression of lung cancer. Through functional analysis of the proximal promoter region of LASP-1, some putative binding sites of transcriptional factors, including Twist2, Nkx2-5 and SOX9 (Fig. 4A), were observed. We amplified the promoter region of LASP-1 (range, $-2,500$ to +1 ) and cloned into pGL3-Basic plasmid. Next, the putative binding sites of Twist2, Nkx2-5 and SOX9 were removed, pGL3-LASP1-mut1, pGL3-LASP1-mut2, and pGL3-LASP1-mut3, respectively. Then, the WT and pGL3LASP1-mut1 constructs were transfected alone or with the pcR-Twist 2 expression vector into HEK293T cells to determine the promoter activities in the absence or presence of Twist2 (Fig. 4B). The data showed that the promoter activity of LASP-1 was markedly increased after transient transfection of HEK293T cells with Twist2 (Fig. 4B, P<0.05). However, the mutated construct significantly decreased the promoting effect of Twist 2 as compared to the control (Fig. 4B). Similar results were observed in Nkx2-5; however, the difference did not reach the statistical significance threshold (Fig. 4C). Furthermore, co-transfection with SOX9 expression vector also showed significantly increased promoter activity of LASP-1 (Fig. 4D, P<0.01). The promoting effect, however, 
A

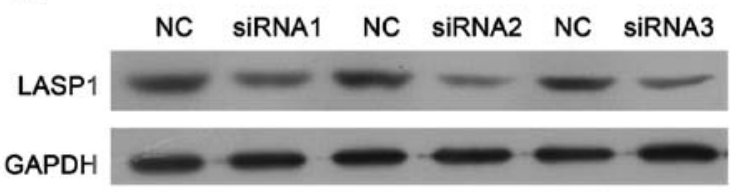

D

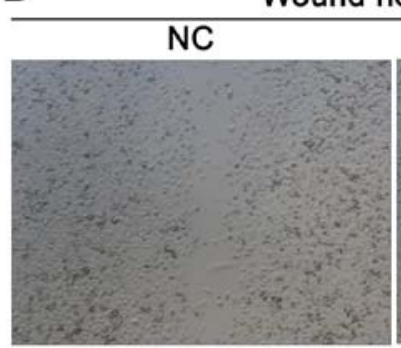

$\mathbf{F}$

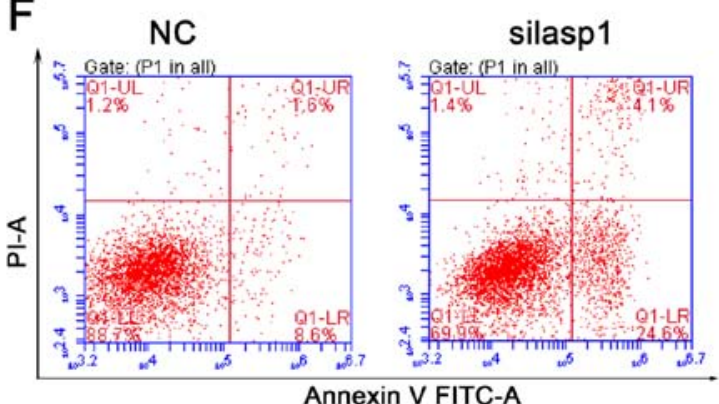

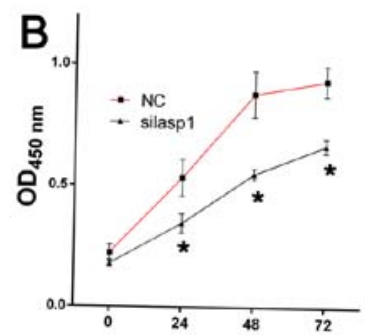

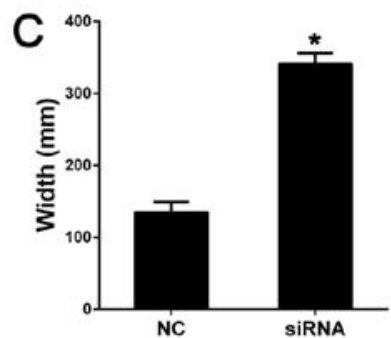

E

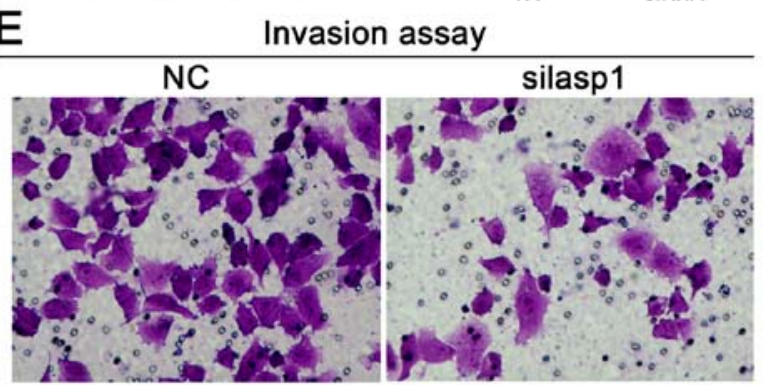

G

NC

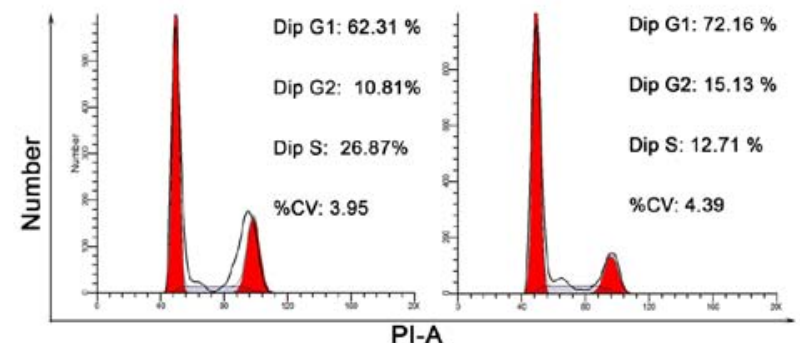

Figure 2. Effects of silencing the expression of LASP-1 in lung cancer cells. (A) NCI-H1650 cells were transfected with negative control (NC), and LASP-1 siRNAs (siRNA1, siRNA2 and siRNA3), respectively. Western blot assay was performed to detect the protein expression levels of LASP-1. GAPDH was used as internal reference. (B) siRNA-mediated silencing of LASP-1 (silasp1) suppressed cell proliferation in NCI-H1650 cells, as determined by CCK-8 assay $(\mathrm{P}<0.05)$. (C and $\mathrm{D})$ Knockdown of the expression of LASP-1 decreased migration in lung cancer cells. All the experiments were performed in triplicates and were repeated thrice; mean $\pm S D$. ${ }^{*} \mathrm{P}<0.05$. (E) Silencing LASP-1 expression impaired cell invasion. Representative images of invaded cells are shown. (F) Cells were stained with PI and Annexin V, and analyzed by flow cytometry. (G) Cell cycle was examined by flow cytometry.

A
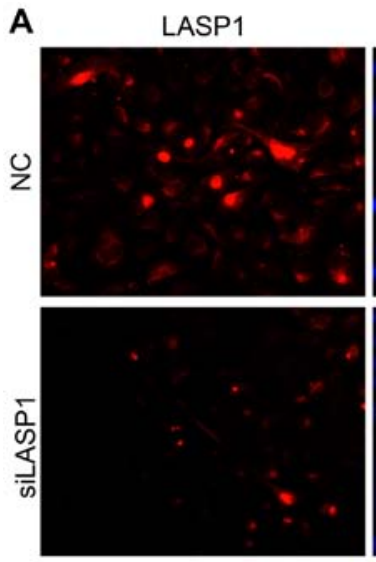

B

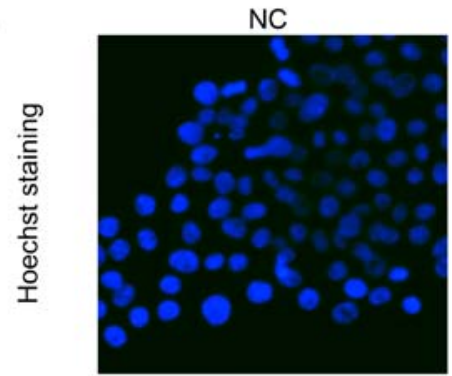

DAPI
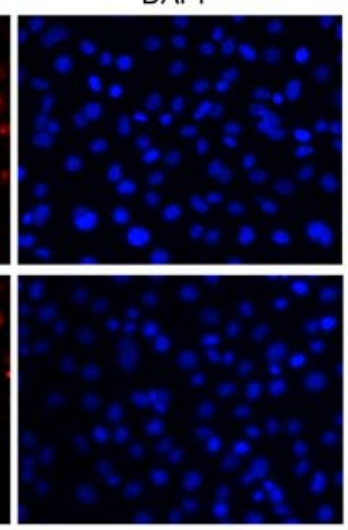

SiLASP1

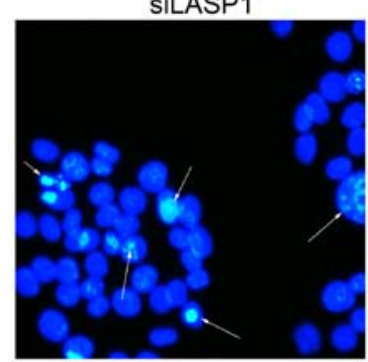

Figure 3. Immunocytochemical staining of LASP-1 in lung cancer cells. (A) Immunofluorescence images of lung cancer cells transfected with siRNA and stained with antibody against LASP-1 (red) and DAPI (blue). (B) Hoechst staining showed that knockdown of the expression of LASP-1 induced cell apoptosis . 


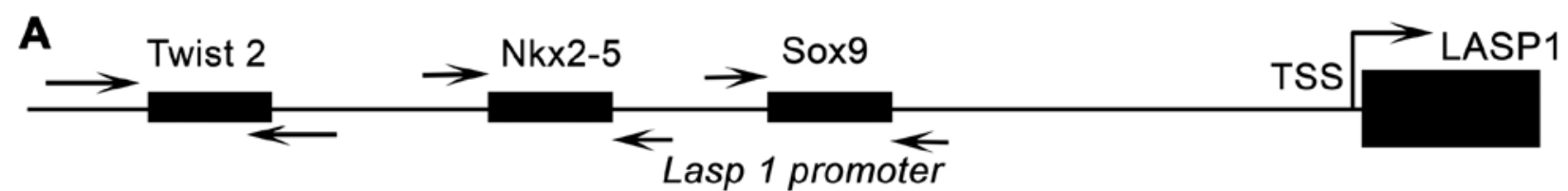

B

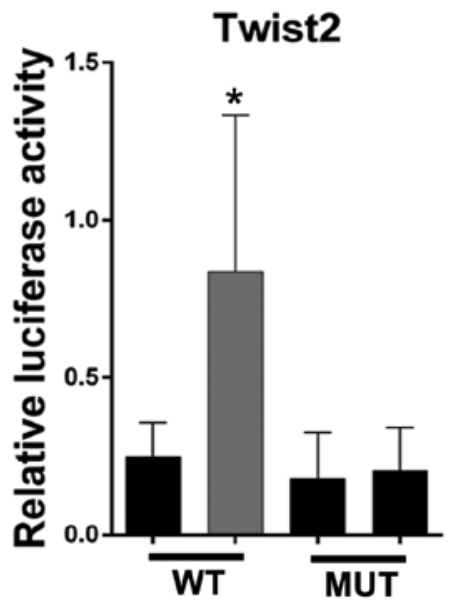

C

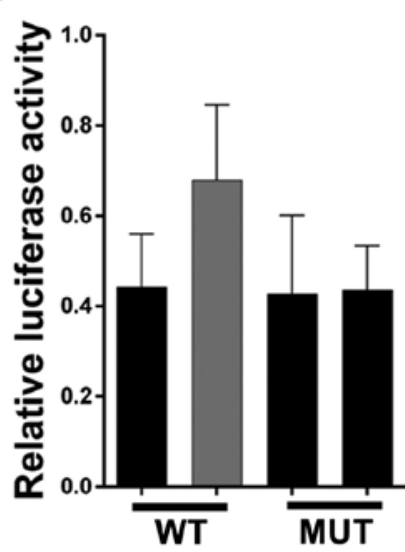

D

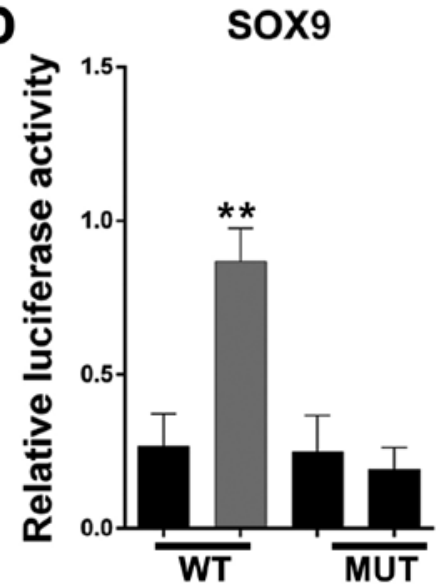

E

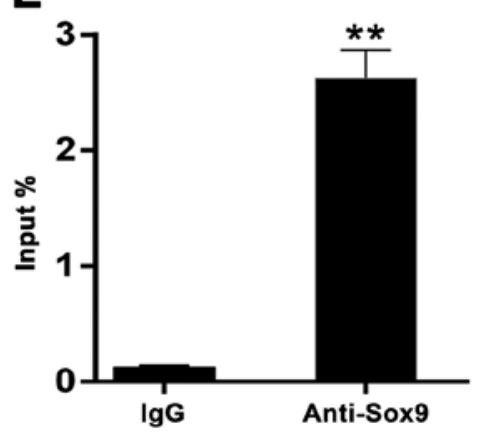

$\mathbf{F}$

G

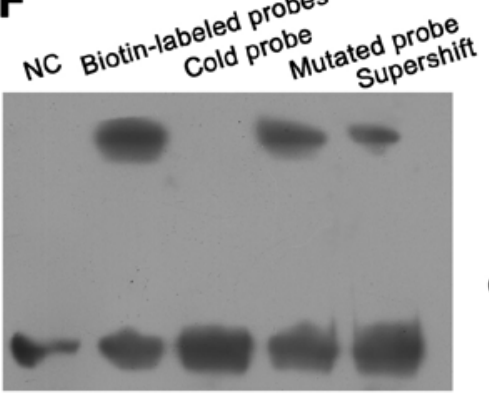
obe

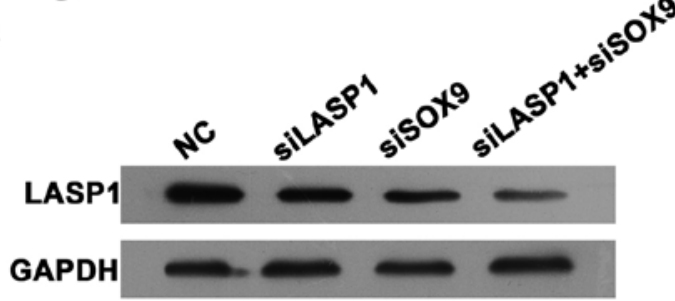

Figure 4. LASP-1 is a direct target gene of SOX9. (A) Map of the LASP-1 promoter region, with the positions of selected consensus binding sites of transcription factors. Primers used to amplify the mutant LASP-1 promoter fragment in the luciferase reporter assays are indicated by an arrow. (B-D) LASP-1 promoter activity after transfection of the WT and mutant promoter constructs alone or together with Twist2, Nkx2-5 and SOX9 expression vectors. The results indicated that SOX9 and Twist 2 significantly enhanced LASP-1 promoter activity. On the contrary, removing the potential binding sites of SOX9 and Twist 2 reverses these promoting effects. The results are expressed as a relative ratio of firefly luciferase to Renilla luciferase. ${ }^{*} \mathrm{P}<0.05 ;{ }^{* *} \mathrm{P}<0.01$. (E) ChIP analysis of $\mathrm{SOX} 9$ at the LASP-1 promoter region in NCI-H1650 cells. The results suggested that SOX9 directly binds to the promoter region of LASP-1. ${ }^{* *} \mathrm{P}<0.01$. (F) EMSA was performed using the nuclear extracts from the treated NCI-H1650 cells. The labeled SOX9 or labeled Mut SOX9 probes were incubated with the nuclear extracts for $10 \mathrm{~min}$. The reaction samples were electrophoresed on a $6 \%$ non-denaturing acrylamide gel. After transferring to the nylon membrane, the biotinlabeled probes were detected. (G) NCI-1650 cells were transfected with negative control (NC), siSOX9, siLASP-1, siSOX9, and siLASP-1. Western blot assay was performed to detect the expression levels of LASP-1. GAPDH was used as internal reference.

can be reversed by deleting the putative binding sites of SOX9 (Fig. 4D). Among the three transcription factors, we focused on SOX9 due to our luciferase reporter assay results. To determine whether LASP-1 is a direct target gene of SOX9, we performed ChIP assays using a monoclonal antibody against SOX9 and amplified the pull-down DNA by real-time PCR. The primers were designed to amplify the region mediating the promoting effects of SOX9 on the LASP-1 promoter. To determine whether SOX9 could directly bind to the LASP-1 element in the promoter region, the electrophoretic mobility shift assay (EMSA) and chromatin immunoprecipitation (ChIP) assays were performed. As shown in Fig. 4E, LASP-1 promoter region was markedly amplified from the SOX9immunoprecipitated NCI-1650 chromatins, but almost absent from chromatin immunoprecipitated by the control IgG. The EMSA showed a DNA/protein band of expected mobility, which reflected the interaction between the probe containing
SOX9 and the nuclear extract of NCI-H1650 cells. The interaction was increased after treatment with biotin-labeled probes, but competitively inhibited by high concentration of cold (unlabeled) probe (not by cold mutated probe; Fig. 4F). In addition, NCI-1650 cells were transfected with negative control (NC), siSOX9, siLASP-1, siSOX9, and siLASP-1. Western blot assay was performed to detect the expression levels of LASP-1. The results showed that LASP-1 expression was decreased in siSOX9 and siLASP-1 groups as compared to NC group; and the combination of siSOX 9 and siLASP-1 has a synergistic effect (Fig. 4G). Taken together, these results indicated that LASP-1 is a direct target gene of SOX9.

Role of LASP-1 in SOX9-induced cell proliferation and invasion. As a transcription activator of LASP-1, SOX9 has been found to be overexpressed in different types of human cancers, including breast cancer and colorectal cancer. Although the 
A

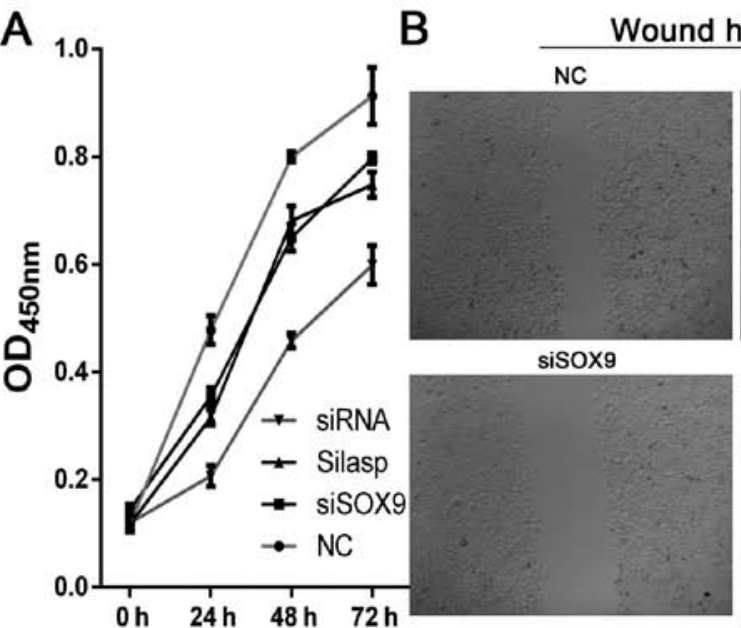

D

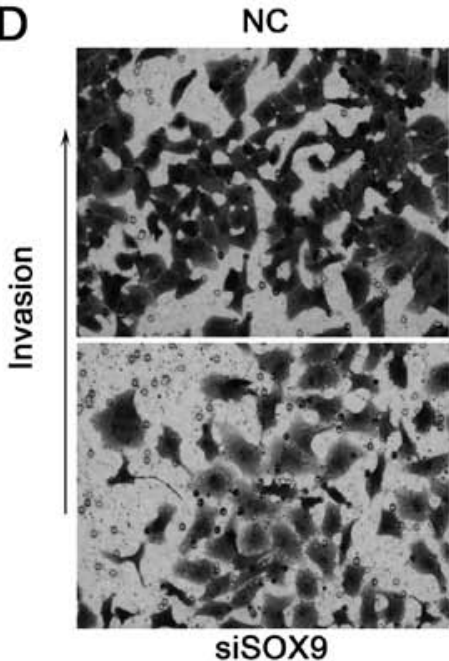

siLASP1

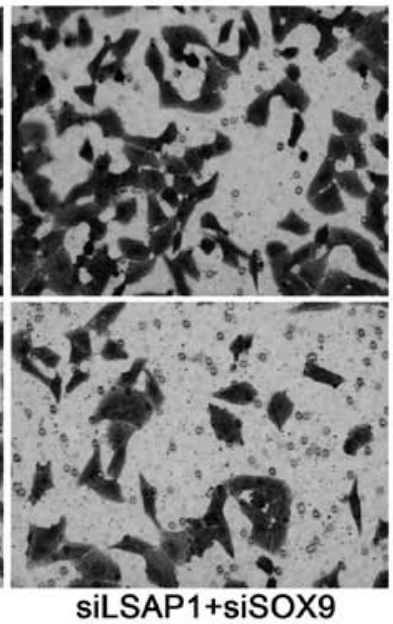

C

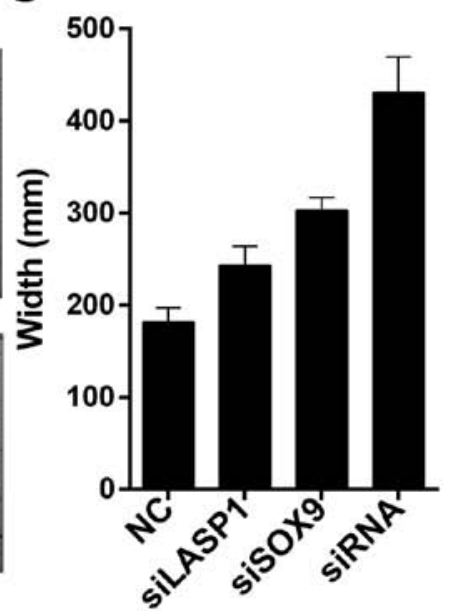

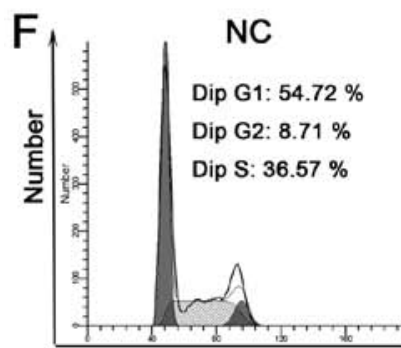

siLASP1
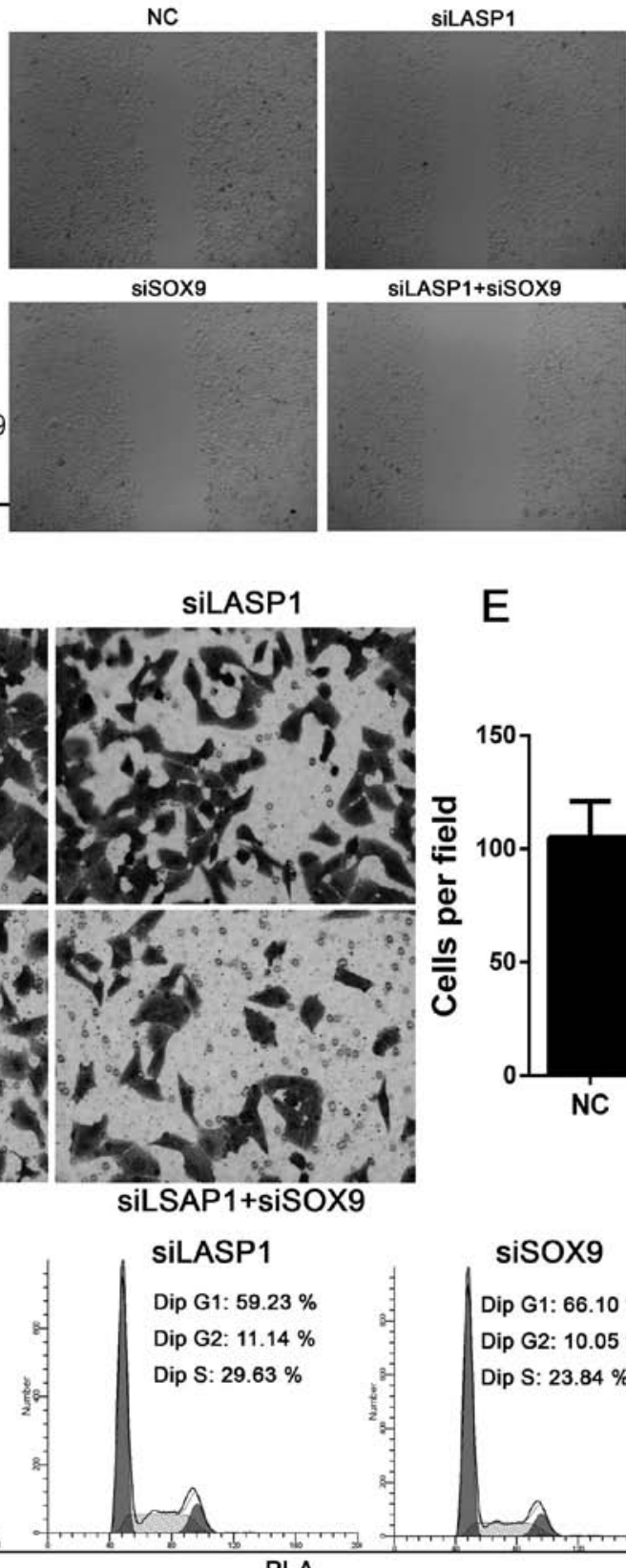

E

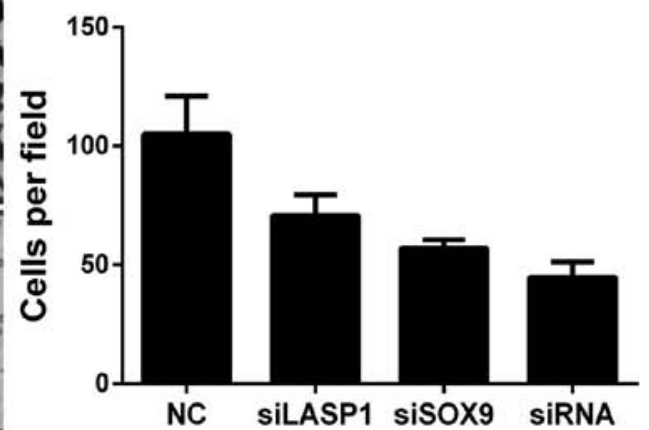

Figure 5. Targeting SOX9-LASP1 axis by siRNA suppressed cell proliferation and invasion. (A) NCI-1650 cells were transfected with negative control (NC), siSOX9, siLASP-1 (Silasp1), siSOX9, and siLASP-1 (siRNA). CCK-8 assays demonstrated that cell proliferation was markedly inhibited in siSOX9 and siLASP-1 cells as compared with control group. (B and C) Cell migration was evaluated by wound healing assay. The results demonstrated that knockdown of the expression of SOX9 reduced cell migration, and combined use of siRNAs targeting SOX9 and LASP-1 significantly inhibited migration in NCI-1650 cells than either used alone. (D and E) Similarly, the Transwell assay showed that the invasive capability of lung cancer cells was decreased after silencing the expression of SOX9 or LASP-1. Combined use of these siRNAs significantly reduced cell invasive ability as compared with NC-transfected cells. (F) Cell cycle distribution was detected by flow cytometry. siRNA in the figure indicates cells transfected with both siSOX9 and siLASP1.

association between the upregulation of SOX9 and lung cancer progression has been reported, the role of SOX9 in regulating cell proliferation and invasion of cancer cells remains in need of further elucidation. To investigate the effect of SOX9, siRNAs were used to silence the expression of SOX9. CCK-8 assays showed that cell proliferation was markedly inhibited in siSOX9 cells as compared to control group. Furthermore, combined use of siRNAs targeting SOX9 and LASP-1 significantly inhibited cell proliferation in NCI-1650 cells than either used alone (Fig. 5A). The scratch assay was used to study the effect of SOX9 on cell migration. The results showed that knockdown of the expression of SOX9 reduced cell migration (Fig. 5B and C). Similarly, the invasive capability of NCI-H1650 cells was decreased after silencing the expression of SOX9. Thus, combined use of siRNAs targeting SOX9 and LASP-1 notably reduced invasive ability as compared with NC-transfected cells (Fig. 5D and 5E). Next, we examined cell cycle distribution using flow cytometry. SOX9 knockdown decreased the proportion of cells in the $S$ phase $(29.63 \%$ of siSOX9 cells vs. $36.57 \%$ of control cells, Fig. 5F), and 
A
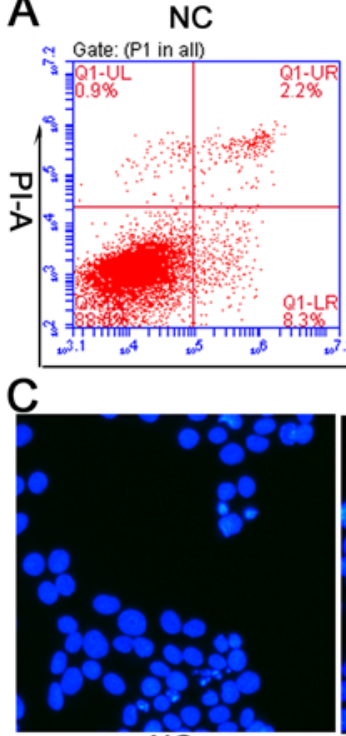

NC
siLASP1

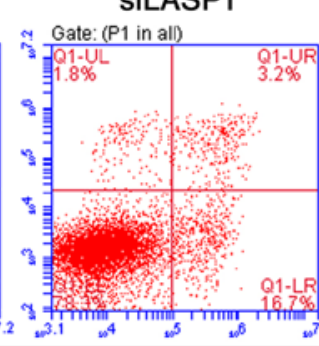

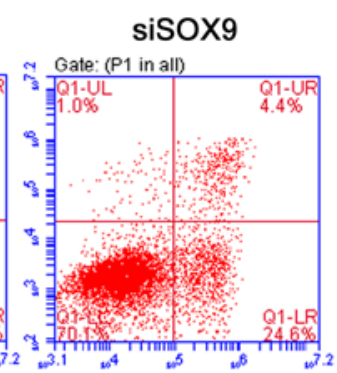

siSOX9

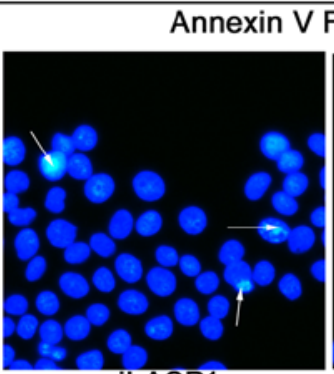

SiLASP1

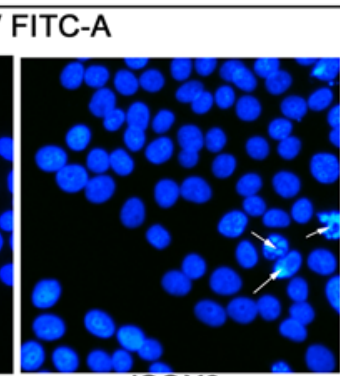

sisoX9
siLASP1+siSOX9
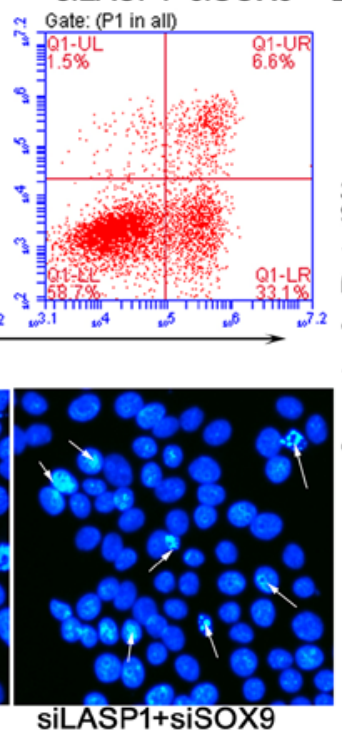

B

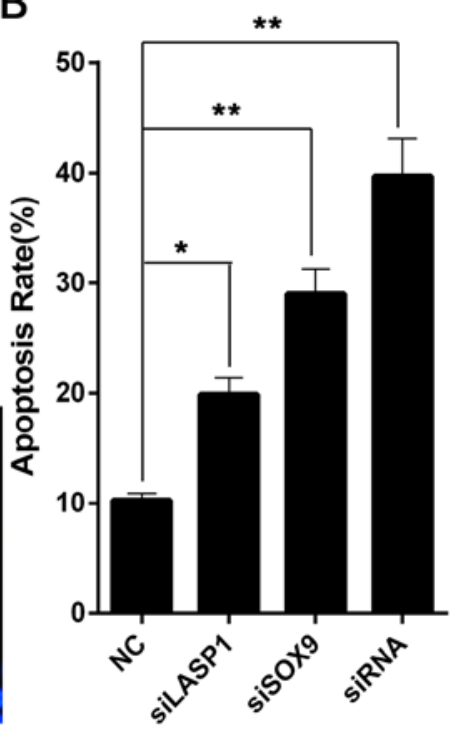

Figure 6. Knockdown of the expression of SOX9 and LASP-1 induced cell apoptosis of lung cancer cells. (A and B) Cell apoptosis was determined by Annexin V and PI staining and analyzed by flow cytometry. The results showed that the combined use of siRNAs targeting SOX9 and LASP-1 induced apoptosis of NCI-1650 cells as compared with control (33.1 vs. 8.3\%). (C) Hoechst staining also showed that cell apoptosis was induced after silencing the expression of SOX9 and LASP-1 in lung cancer cells. Authors: *** please describe.

increased the proportion of cells in the G1 phase as compared with control (Fig. 5F). In addition, combined use of siRNAs targeting SOX9 and LASP-1 induced apoptosis of NCI-H1650 cells as compared with control (33.1 vs. 8.3\%, Fig. 6A and B). Hoechst staining also showed that cell apoptosis was increased after silencing the expression of SOX9 and LASP-1 (Fig. 6C). These results showed that SOX9-LASP-1 axis was involved in the progression of lung cancer.

\section{Discussion}

Various studies have shown that LASP-1 is overexpressed in many cancers, such as metastatic breast cancer, ovarian cancer, and colorectal cancer $(18,34,35)$. Its expression is strongly associated with lymph node metastasis and poor clinical prognosis. However, until now, little is known about the role of LASP-1 in lung cancer progression. In this study, results from real-time PCR and immunohistochemical assays demonstrated that LASP-1 was not expressed in tumor-adjacent normal tissues; however, significant expression was observed in lung cancer tissues, which was consistent with the results reported by Zheng et al (7).

In this study, the effect of LASP-1 on lung cancer pathogenesis was evaluated in vitro using NCI-H1650 cells. Silencing LASP-1 by siRNA transfection significantly inhibited cell proliferation and induced cell apoptosis. Furthermore, the results of wound healing and Transwell assays indicated that LASP-1 silencing suppressed the migration and invasion of lung cancer cells. These findings are in line with several other studies demonstrating reduced cell motility after LASP-1 silencing (17-19,36). However, the studies focused on the underlying molecular mechanism of LASP-1 in promoting tumor progression are very rare. Grunewald $e t$ al and Shimizu et al reported that downregulation of LASP-1 induced G2/M phase accumulation of breast cancer and ovarian cancer cells $(17,37)$. In addition, silencing LASP-1 expression resulted in an increase in number of cells in G1 phase. In addition, our study showed that knockdown of LASP-1 expression induced G2 phase accumulation; besides, knockdown of LASP-1 leads to decreased expression of cyclin A and cyclin B, and increased phospho-cdc2 (Tyr15) expression (37). Our study demonstrated that silencing of LASP1 significantly inhibited prostate cancer cell growth by decreasing cyclin D1 and increasing $\mathrm{p} 21$ and p27 (38). Migration and invasion are key determinants of cancer cell progression and metastasis. Some studies have demonstrated that LASP-1 is involved in cell migration and invasion, based on the ability of the cells to interact with a series of focal adhesion proteins, such as F-actin, zyxin, and CXCR2 (18,39). Epithelial-mesenchymal transition (EMT) is a reversible process by which cancer cells can switch from a sessile epithelial phenotype to an invasive mesenchymal state (40). In 2016, Zhang et al reported that knockdown of LASP-1 expression inhibited the migration and invasion of CCA cells by inducing EMT (20). Wang et al showed that LASP-1 plays a critical role in the TGF- $\beta$-mediated EMT process in colorectal cancer metastasis (35). While increasing number of evidence suggests that LASP-1 plays an important role in cancer progression, the role of LASP-1 in mediating the proliferation and metastasis of cancer cells remains unclear and requires further study.

An additional observation further underscores the importance of LASP-1 in cancer. Previous studies have shown that upstream regulatory factors, HIF-1 and p53, have critical functions in regulating LASP-1 expression $(41,42)$. The expression of LASP-1 in PDAC cells was activated by HIF-1 $\alpha$ by direct binding to hypoxia response element in the LASP-1 promoter (42). On the contrary, transcription factor, p53, repressed LASP-1 expression (20). Furthermore, 
IGF-1-induced expression of LASP-1 gene in MCF-7 cells was described in a recent study (43). Only few studies have explored the regulatory mechanism of LASP-1 in lung cancer. In this study, we demonstrated for the first time that SOX9 induced LASP-1 expression. Dual luciferase reporter assay and ChIP assays demonstrated that LASP-1 is a direct target gene of SOX9. A series of studies have demonstrated that SOX9 is a multifaceted transcription factor, which is involved in the development of numerous organ and tissues. SOX9 is reported to be upregulated in several types of human cancers, including lung ADC (30). Depletion of LASP-1 by specific siRNA inhibited lung cancer cell proliferation and invasion. Moreover, combined use of siRNAs targeting SOX9 and LASP-1 significantly inhibited cell proliferation, migration, and invasion of NCI-H1650 cells. These findings suggested that SOX9-LASP1 axis is involved in lung cancer cell progression.

In conclusion, our results demonstrated that LASP-1 was upregulated in lung cancer and played an important role in cell proliferation, migration, and invasion. Mechanistic analysis identified LASP-1 as a novel direct target of SOX9. These findings suggested that LASP-1 is a promising therapeutic target, and targeting SOX9-LASP1 axis may be an effective method for treatment of lung cancer.

\section{References}

1. Ferlay J, Shin HR, Bray F, Forman D, Mathers C and Parkin DM: Estimates of worldwide burden of cancer in 2008: GLOBOCAN 2008. Int J Cancer 127: 2893-2917, 2010.

2. Ferlay J, Soerjomataram I, Dikshit R, Eser S, Mathers C, Rebelo M, Parkin DM, Forman D and Bray F: Cancer incidence and mortality worldwide: Sources, methods and major patterns in GLOBOCAN 2012. Int J Cancer 136: E359-E386, 2015.

3. Dela Cruz CS, Tanoue LT and Matthay RA: Lung cancer: Epidemiology, etiology, and prevention. Clin Chest Med 32: 605-644, 2011.

4. Steliga MA and Dresler CM: Epidemiology of lung cancer: Smoking, secondhand smoke, and genetics. Surg Oncol Clin N Am 20: 605-618, 2011.

5. Yano T, Haro A, Shikada Y, Maruyama R and Maehara Y: Non-small cell lung cancer in never smokers as a representative 'non-smoking-associated lung cancer': Epidemiology and clinical features. Int J Clin Oncol 16: 287-293, 2011.

6. Villar Álvarez F, Muguruza Trueba I and Vicente Antunes SI: Notes on recurrence and second tumors in lung cancer. Arch Bronconeumol 52: 545-546, 2016.

7. Zheng J, Wang F, Lu S and Wang X: LASP-1, regulated by miR-203, promotes tumor proliferation and aggressiveness in human non-small cell lung cancer. Exp Mol Pathol 100: 116-124, 2016.

8. Siegel R, Ma J, Zou Z and Jemal A: Cancer statistics, 2014. CA Cancer J Clin 64: 9-29, 2014.

9. Temel JS, Greer JA, Muzikansky A, Gallagher ER, Admane S, Jackson VA, Dahlin CM, Blinderman CD, Jacobsen J, Pirl WF, et al: Early palliative care for patients with metastatic non-smallcell lung cancer. N Engl J Med 363: 733-742, 2010.

10. Tomasetto C, Moog-Lutz C, Régnier CH, Schreiber V, Basset P and Rio MC: Lasp-1 (MLN 50) defines a new LIM protein subfamily characterized by the association of LIM and SH3 domains. FEBS Lett 373: 245-249, 1995.

11. Orth MF, Cazes A, Butt E and Grunewald TG: An update on the LIM and SH3 domain protein 1 (LASP1): A versatile structural, signaling, and biomarker protein. Oncotarget 6 : 26-42, 2015

12. Segerer SE, Bartmann C, Kaspar S, Müller N, Kapp M, Butt E and Kämmerer U: The cytoskeletal protein LASP-1 differentially regulates migratory activities of choriocarcinoma cells. Arch Gynecol Obstet 293: 407-414, 2016.

13. Rachlin AS and Otey CA: Identification of palladin isoforms and characterization of an isoform-specific interaction between Lasp-1 and palladin. J Cell Sci 119: 995-1004, 2006.
14. Grunewald TG and Butt E: The LIM and SH3 domain protein family: Structural proteins or signal transducers or both? Mol Cancer 7: 31, 2008.

15. Duvall-Noelle N, Karwandyar A, Richmond A and Raman D: LASP-1: A nuclear hub for the UHRF1-DNMT1-G9a-Snail1 complex. Oncogene 35: 1122-1133, 2016.

16. Frietsch JJ, Grunewald TG, Jasper S, Kammerer U, Herterich S, Kapp M, Honig A and Butt E: Nuclear localisation of LASP-1 correlates with poor long-term survival in female breast cancer. Br J Cancer 102: 1645-1653, 2010.

17. Grunewald TG, Kammerer U, Schulze E, Schindler D, Honig A, Zimmer $M$ and Butt E: Silencing of LASP-1 influences zyxin localization, inhibits proliferation and reduces migration in breast cancer cells. Exp Cell Res 312: 974-982, 2006.

18. Grunewald TG, Kammerer U, Winkler C, Schindler D, Sickmann A, Honig A and Butt E: Overexpression of LASP-1 mediates migration and proliferation of human ovarian cancer cells and influences zyxin localisation. Br J Cancer 96: 296-305, 2007.

19. Zheng J, Yu S, Qiao Y, Zhang H, Liang S, Wang H, Liu Y, Zhou F, Jiang $\mathbf{J}$ and Lu S: LASP-1 promotes tumor proliferation and metastasis and is an independent unfavorable prognostic factor in gastric cancer. J Cancer Res Clin Oncol 140: 1891-1899, 2014

20. Zhang H, Li Z, Chu B, Zhang F, Zhang Y, Ke F, Chen Y, Xu Y, Liu S, Zhao S, et al: Upregulated LASP-1 correlates with a malignant phenotype and its potential therapeutic role in human cholangiocarcinoma. Tumour Biol 37: 8305-8315, 2016.

21. Zhao L, Wang H, Liu C, Liu Y, Wang X, Wang S, Sun X, Li J, Deng Y, Jiang Y, et al: Promotion of colorectal cancer growth and metastasis by the LIM and SH3 domain protein 1. Gut 59: 1226-1235, 2010

22. Lin X, Liu X, Fang Y and Weng X: LIM and SH3 protein 1 promotes tumor proliferation and metastasis in lung carcinoma. Oncol Lett 12: 4756-4760, 2016.

23. Yang F, Zhou X, Du S, Zhao Y, Ren W, Deng Q, Wang F and Yuan J: LIM and SH3 domain protein 1 (LASP-1) overexpression was associated with aggressive phenotype and poor prognosis in clear cell renal cell cancer. PLoS One 9: e100557, 2014.

24. Akiyama H, Chaboissier MC, Martin JF, Schedl A and de Crombrugghe B: The transcription factor Sox9 has essential roles in successive steps of the chondrocyte differentiation pathway and is required for expression of Sox 5 and Sox6. Genes Dev 16: 2813-2828, 2002.

25. Spokony RF, Aoki Y, Saint-Germain N, Magner-Fink E and Saint-Jeannet JP: The transcription factor Sox9 is required for cranial neural crest development in Xenopus. Development 129: 421-432, 2002.

26. Chaboissier MC, Kobayashi A, Vidal VI, Lützkendorf S, van de Kant HJ, Wegner M, de Rooij DG, Behringer RR and Schedl A: Functional analysis of Sox8 and Sox9 during sex determination in the mouse. Development 131: 1891-1901, 2004.

27. Müller P, Crofts JD, Newman BS, Bridgewater LC, Lin CY, Gustafsson JA and Ström A: SOX9 mediates the retinoic acidinduced HES-1 gene expression in human breast cancer cells. Breast Cancer Res Treat 120: 317-326, 2010.

28. Qin GQ, He HC, Han ZD, Liang YX, Yang SB, Huang YQ, Zhou L, Fu H, Li JX, Jiang FN, et al: Combined overexpression of HIVEP3 and SOX9 predicts unfavorable biochemical recurrence-free survival in patients with prostate cancer. Onco Targets Ther 7: 137-146, 2014.

29. Bruun J, Kolberg M, Nesland JM, Svindland A, Nesbakken A and Lothe RA: Prognostic significance of $\beta$-catenin, E-cadherin, and SOX9 in colorectal cancer: Results from a large populationrepresentative series. Front Oncol 4: 118, 2014.

30. Jiang SS, Fang WT, Hou YH, Huang SF, Yen BL, Chang JL, Li SM, Liu HP, Liu YL, Huang CT, et al: Upregulation of SOX9 in lung adenocarcinoma and its involvement in the regulation of cell growth and tumorigenicity. Clin Cancer Res 16: 4363-4373, 2010.

31. Wang X, Ju Y, Zhou MI, Liu X and Zhou C: Upregulation of SOX9 promotes cell proliferation, migration and invasion in lung adenocarcinoma. Oncol Lett 10: 990-994, 2015.

32. Wang X, Liu Y, Liu X, Yang J, Teng G, Zhang L and Zhou C: MiR-124 inhibits cell proliferation, migration and invasion by directly targeting SOX9 in lung adenocarcinoma. Oncol Rep 35: 3115-3121, 2016.

33. Zhou CH, Ye LP, Ye SX, Li Y, Zhang XY, Xu XY and Gong LY: Clinical significance of SOX9 in human non-small cell lung cancer progression and overall patient survival. J Exp Clin Cancer Res 31: 18, 2012. 
34. Grunewald TG, Kammerer U, Kapp M, Eck M, Dietl J, Butt E and Honig A: Nuclear localization and cytosolic overexpression of LASP-1 correlates with tumor size and nodal-positivity of human breast carcinoma. BMC Cancer 7: 198, 2007.

35. Wang H, Shi J, Luo Y, Liao Q, Niu Y, Zhang F, Shao Z, Ding Y and Zhao L: LIM and SH3 protein 1 induces TGF $\beta$-mediated epithelial-mesenchymal transition in human colorectal cancer by regulating S100A4 expression. Clin Cancer Res 20: 5835-5847, 2014.

36. Hailer A, Grunewald TG, Orth M, Reiss C, Kneitz B, Spahn M and Butt E: Loss of tumor suppressor mir-203 mediates overexpression of LIM and SH3 protein 1 (LASP1) in high-risk prostate cancer thereby increasing cell proliferation and migration. Oncotarget 5: 4144-4153, 2014.

37. Shimizu F, Shiiba M, Ogawara K, Kimura R, Minakawa Y, Baba T, Yokota S, Nakashima D, Higo M, Kasamatsu A, et al: Overexpression of LIM and SH3 protein 1 leading to accelerated $\mathrm{G} 2 / \mathrm{M}$ phase transition contributes to enhanced tumourigenesis in oral cancer. PLoS One 8: e83187, 2013.

38. Dejima T, Imada K, Takeuchi A, Shiota M, Leong J, Tombe T, Tam K, Fazli L, Naito S, Gleave ME, et al: Suppression of LIM and SH3 domain protein 1 (LASP1) negatively regulated by androgen receptor delays castration resistant prostate cancer progression. Prostate 77: 309-320, 2017.
39. Raman D, Sai J, Neel NF, Chew CS and Richmond A: LIM and SH3 protein-1 modulates CXCR2-mediated cell migration. PLoS One 5: e10050, 2010

40. Felipe Lima J, Nofech-Mozes S, Bayani J and Bartlett JM: EMT in breast carcinoma-A review. J Clin Med 5: E65, 2016.

41. Wang B, Feng P, Xiao Z and Ren EC: LIM and SH3 protein 1 (Lasp1) is a novel p53 transcriptional target involved in hepatocellular carcinoma. J Hepatol 50: 528-537, 2009.

42. Zhao T, Ren H, Li J, Chen J, Zhang H, Xin W, Sun Y, Sun L, Yang Y, Sun J, et al: LASP1 is a HIFla target gene critical for metastasis of pancreatic cancer. Cancer Res 75: 111-119, 2015.

43. Loughran G, Huigsloot M, Kiely PA, Smith LM, Floyd S, Ayllon V and O'Connor R: Gene expression profiles in cells transformed by overexpression of the IGF-I receptor. Oncogene 24: 6185-6193, 2005 . 